\title{
Why Pashmina Goat Produces Long Hair-fiber and Barbari doesn't: A Differential Gene Expression Study
}

\author{
Rashid Saif ${ }^{1,2}$, Tania Mahmood ${ }^{2}$, Aniqa Ejaz ${ }^{2}$, Saeeda $\mathrm{Zia}^{3}$
}

${ }^{1}$ Institute of Biotechnology, Gulab Devi Educational Complex, Lahore, Pakistan

${ }^{2}$ Decode Genomics, Punjab University Employees Housing Scheme (II), Lahore, Pakistan

${ }^{3}$ Department of Sciences and Humanities, National University of Computer and Emerging Sciences, Lahore, Pakistan

Corresponding author: rashid.saif37@gmail.com

\begin{abstract}
The Pashmina and Barbari are two famous goat breeds found in the wide areas of the Indo-Pak region. Pashmina is famous for its long hair-fiber (Cashmere) production while Barbari is not-selected for this trait. So, the mRNA expression profiling in the skin samples of both breeds would be an attractive and judicious approach for detecting putative genes involved in this valued trait. Here, we performed differential gene expression analysis on publicly available RNA-Seq data from both breeds. Out of 44,617,994 filtered reads of Pashmina and 55,995,999 of Barbari which are $76.48 \%$ and $73.69 \%$ mapped to the ARS1 reference transcriptome assembly respectively. A Pairwise comparison of both breeds resulted in 47,159 normalized expressed transcripts while 8,414 transcripts are differentially expressed above the significant threshold. Among these, 4,788 are upregulated in Pashmina while 3,626 transcripts are upregulated in Barbari. Fifty-nine transcripts harbor 57 genes including 32 LOC genes and 24 are annotated genes which were selected on the basis of TMM counts $>500$. Genes with ectopic expressions other than uncharacterized and LOC symbol genes are Keratins (KRT) and Keratin Associated Proteins (KRTAPS), CystatinA\&6, TCHH, SPRR4, PPIA, SLC25A4, S100A11, DMKN, LOR, ANXA2, PRR9 and SFN. All of these genes are likely to be involved in keratinocyte differentiation, sulfur matrix proteins, dermal papilla cells, hair follicles proliferation, hair curvature, wool fiber diameter, hair transition, hair shaft differentiation and its keratinization. These differentially expressed reported genes are critically valuable for enhancing the quality and quantity of the pashmina fiber and overall breed improvement. This study will also provide important information on hair follicle differentiation for further enrichment analyses and introducing this valued trait to other goat breeds as well.
\end{abstract}

Key words: Transcriptome analysis, Capra hircus, Differential gene expression, Pashmina goat, Barbari goat, RNA-seq

\section{Introduction}

Hair follicles (HFs) are composed of integumental tissues which consist of outer dermal and inner epidermal layer cells. Their interaction initiates the hair cycle composed of anagen (proliferation), telogen (resting), catagen (regression), exogen (shedding) and kinogen (new growth) phases that ultimately results in keratinized end products including hairs, skin, horns etc. [1]. It is well recognized that the contribution of animal's hair in thermal insulation and visual display affect the human commerce for which they harvest the fiber [2]. Commercially valuable fiber produced are of goats that usually consist of double coat, the outer long hairs made up of primary hair follicles (PHFs) while the secondary hair follicles (SHFs) make up the inner wool coat (the textile fiber cashmere). The under wool coat is $<19 \mu \mathrm{m}$ in diameter that is obtained mainly from Pashmina goat breed [3]. The fitness of pashmina fiber depends on number and small diameter of SHFs. It is of prime significance to the country's economy as it is globally valued for its warmth, fine, soft and thick fiber. In the past for centuries, this Pashmina goat have roamed in western zones of Tibetan plateau and now it habitats the high altitude of Himalayas in Pakistan, Nepal and Northern India [4]. As a functional mini-organ, the molecular mechanisms controlling HFs in indigenous livestock are now addressed widely. RNA-Seq technology due to its cost-effectiveness provides such platform to elucidate the novel transcripts, transcripts with low or high abundance and differential gene expression among various breeds. This including other high throughput sequencing technologies can also provide a detailed and accurate molecular insight of the organism under consideration for which the genomic resources are limited.

Herein, we focused to analyze the publically available datasets of Pashmina and Barbari goat's transcriptome skin sample. In contrast to Pashmina goat breed, Barbari is small, short-haired, meat and milk yielding breed and is indigenous to Pakistan, Nepal, India, Mauritius and Vietnam [5]. We compared the digital transcript expression profiles from both breeds to look for highly expressed genes related to HFs. 


\section{Materials and Methods}

\subsection{Sample source and quality analysis}

Whole transcriptome skin sample of Pashmina and Barbari goats were selected to analyze the differential expression of wool/fiber producing genes. Both breeds are shown in Figure 1.

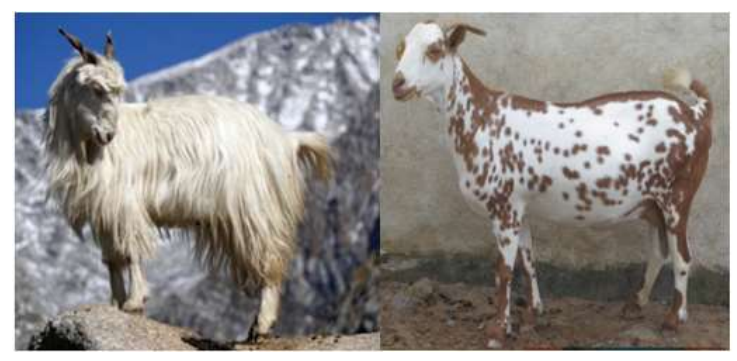

Figure 1. Pashmina goat (left) [6] vs. Barbari (right) [7].

Sequencing reads were taken from ENA source under the experiment accession: SRX3414248 and SRX6655258 respectively. Prior to mapping and quantification of paired end fastq sequences, files were first subjected to quality checks using FastQC (v0.11.8) software [8]. Only the clean reads were then retained by filtering low quality bases by Trimmomatic (v0.36) using sliding window approach [9].

\subsection{Normalization of quantified expression levels}

We calculated number of mapped reads to each transcript directly from filtered reads by Salmon tool [10] in mapping based mode providing Capra hircus transcriptome reference fasta file to perform mapping as an intermediate step. The normalization of raw counts using Trinity's tool script [11], abundance_extimates_to_matrix.pl reported TMM (Trimmed Mean of M-values) and TPM (Transcripts Per Million) values which are effective to eliminate sequencing and gene length biases.

\subsection{Gene expression profiling and visualization}

The differential expression in transcripts of Pashmina and Barbari goat's skin sample was analyzed to figure out the expression of key genes related to wool production. The number of expressed transcripts in TPM normalized matrix was counted using count_matrix_features_given_MIN_TPM_threshold.pl script of Trinity [12] and then used plot function on $\mathrm{R}$ for visual inference. Pairwise comparison between the two samples using counts matrix identified DE transcripts by running script run_DE_analysis.pl. The parameter method was set to edgeR and dispersion value 0.1 . This generated MA and volcano plots. From TMM matrix, differentially expressed transcripts (DETs) were extracted and clustered using analyze_diff_exp.pl script. Transcripts were considered significant that have $\mathrm{p}$ value at most 0.001 and $\log 2$ fold-change $(\log 2 \mathrm{FC})$ of 2 . Heatmap and correlation graph were also generated [13].

\section{Results}

\subsection{Quality checks and mapping}

Trimming of 47,830,768 reads of Pashmina breed resulted in survival of 44,617,994 (93.28\%) pair of reads while from a total of 55,995,999 reads of Barbari 55,502,832 (99.12\%) pair of reads retained after cleaning of low quality bases. Overall GC\% of both breeds is 52\%. Quality scores graphs is shown in (Figure 2). In addition, 76.48\% (34126799 reads) of Pashmina breed and 73.69\% (40902778 reads) of Barbari mapped successfully to the reference ARS1transcriptome. 
(a)

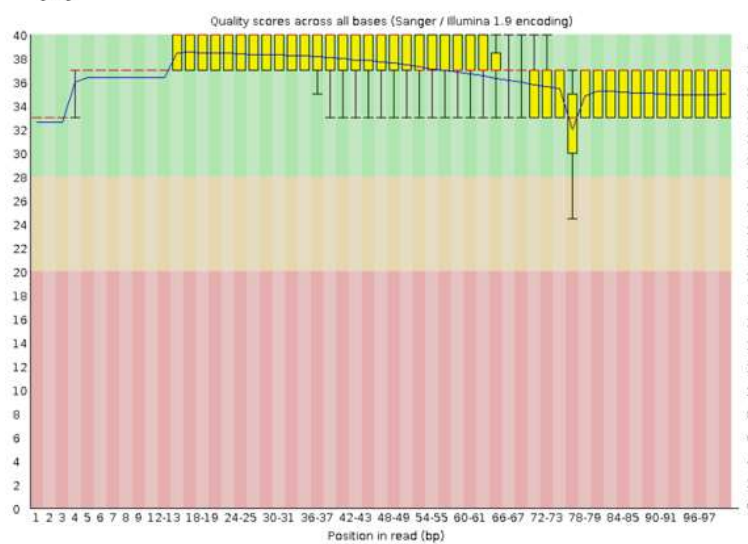

(b)

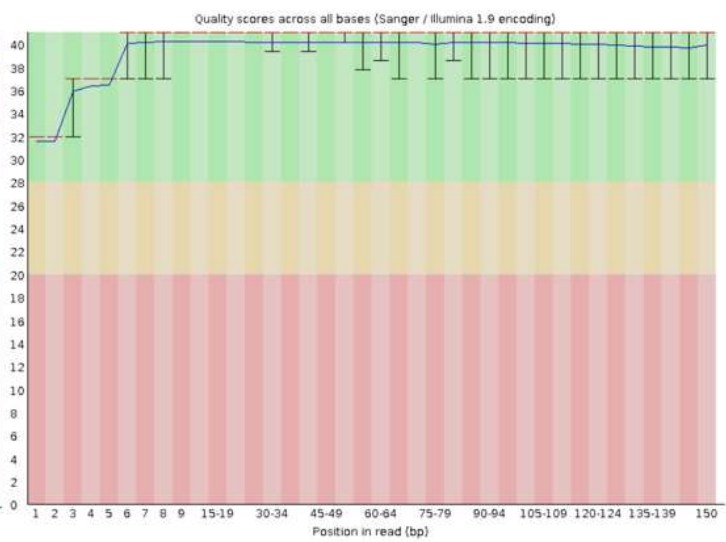

Figure 2. Graphical output of FastQC report showing the quality scores across all bases. (a) Pashmina breed. (b) Barbari breed. The yellow bar exhibits interquartile range, red line is for median value while blue line signifies mean quality of bases.

\subsection{Identification and screening of expressed transcripts}

To detect the key genes related to cashmere production in Pashmina and Barbari, the pairwise comparison of both breeds was carried out. On the basis of TPM matrix maximally, 47,159 transcripts are found to be expressed. (Figure 3) displays the distribution of these expressed transcripts as MA and Volcano plots.

(a)

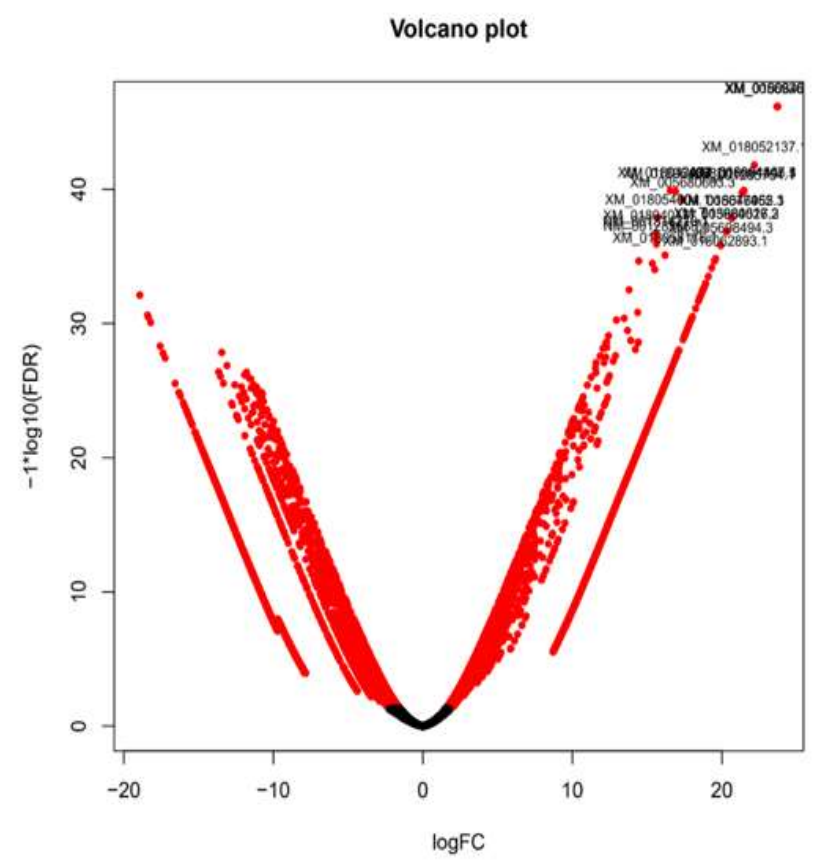

(b)

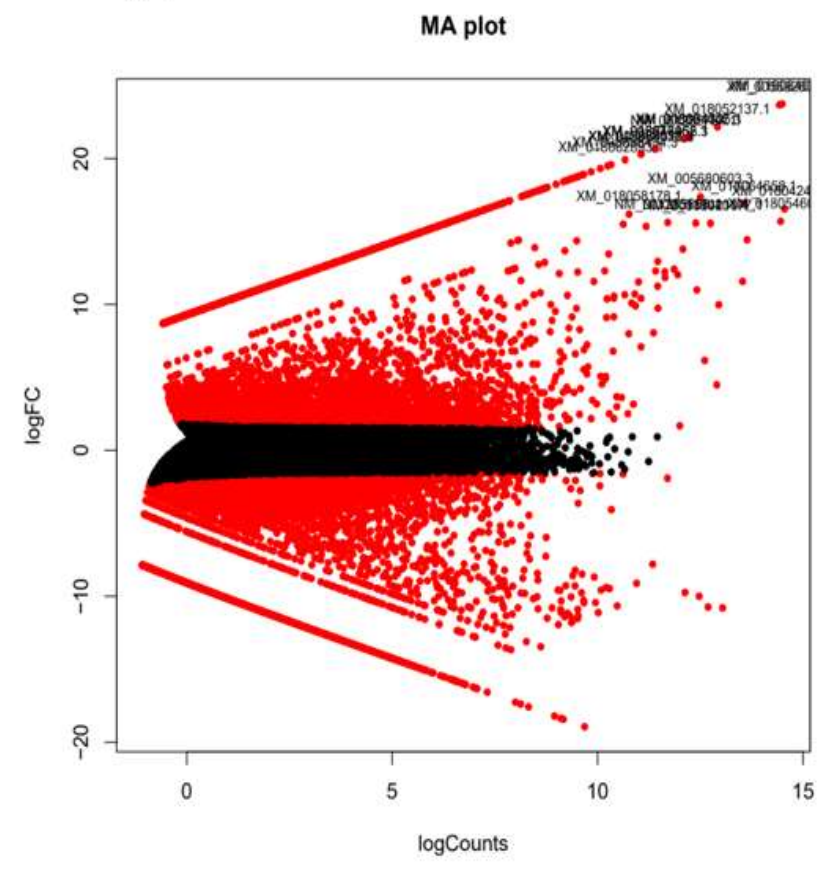

Figure 3. Distribution of transcripts is displayed. (a) Volcano plot (b) MA plot. Non-significant transcripts are represented with black dots. The red dots on right side in volcano plot and upper one in MA plot are upregulated transcripts. Top most genes are annotated with transcript IDs.

While calculating the DETs at a significant threshold (p-value cutoff for FDR $<0.001$ and $\log 2 \mathrm{FC}>2$ ), we found 8,414 transcripts that appeared to be differentially expressed between Pashmina and Barbari (Table S1). 
Among the DETs, 4,788 are upregulated in Pashmina (Table S2) while 3,626 transcripts are upregulated in Barbari breed (Table S3).

\subsection{High differential expression of HF genes}

Two-dimensional hierarchical clustering of differentially expressed transcripts was performed to group the similar ones based on the expression patterns which classified the differentially expressed features on a heatmap as shown in (Figure 4).

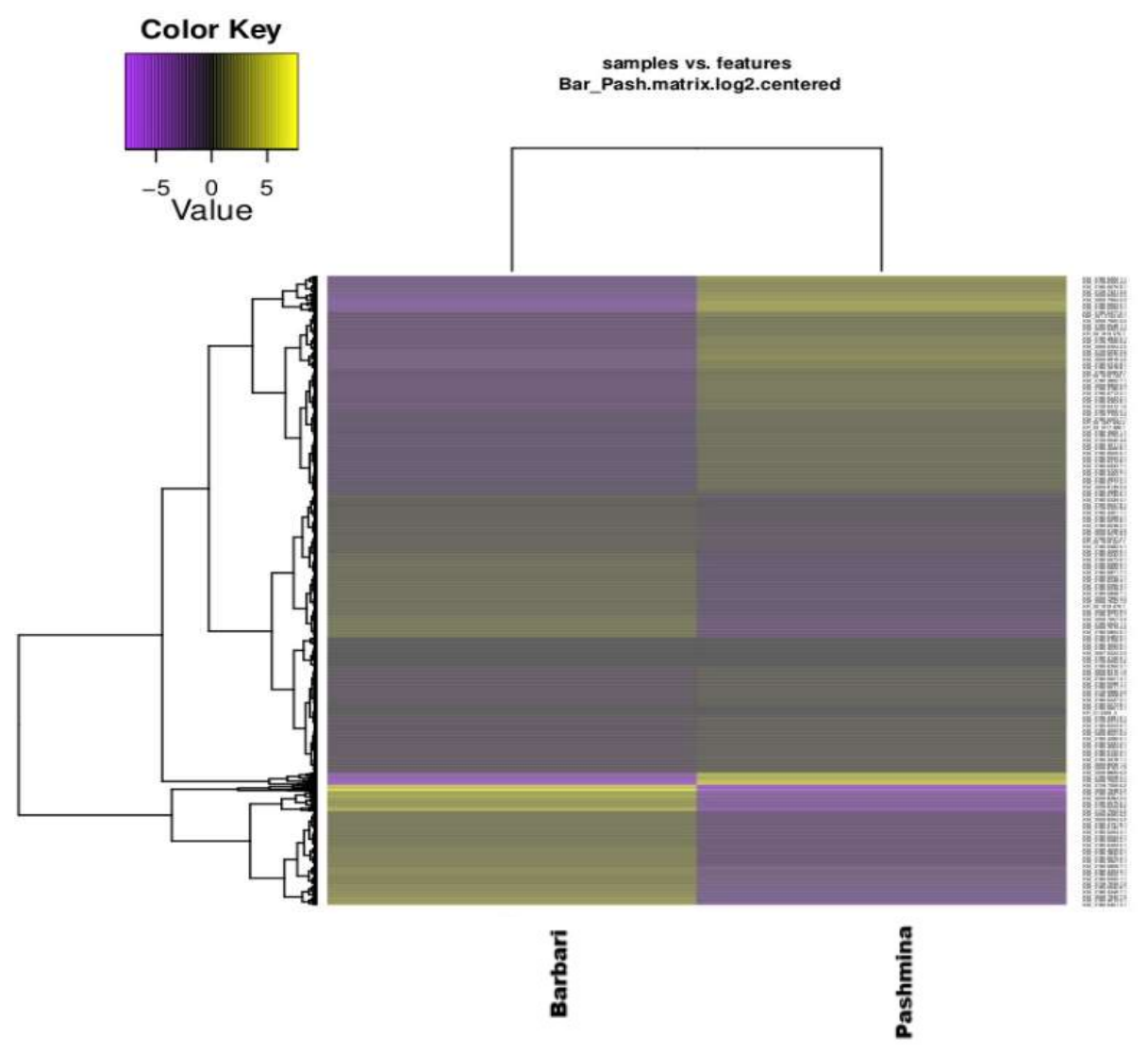

Figure 4. Hierarchical clustering of DETs in Pashmina and Barbari goat based on TMM expressions on heatmap. The upregulated expression of transcripts are shown in yellow while down-regulated transcripts are represented in purple color.

The transcripts with the highest expression in Pashmina and Barbari were separately analyzed to look for the genes involved in wool production. A high expression level having the average normalized counts $>500$ was selected to single out the most expressed genes related to HFs that divulged 59 transcripts consisting of 57 genes including 32 LOC symbol genes and 24 known genes. Table 1 detailed the known genes that have role in keratinization pathway, hair development or morphogenesis, fiber diameter etc. 
Table 1. Fold Changes of DEGs responsible for HFs in both breeds

\begin{tabular}{|c|c|c|c|c|c|}
\hline Transcript ID & Genes & Gene names & $\operatorname{LogFC}$ & $\begin{array}{l}\text { Pashmina } \\
\text { TMM counts }\end{array}$ & $\begin{array}{l}\text { Barbari } \\
\text { TMM counts }\end{array}$ \\
\hline XM 005692310.3 & DMKN & Dermokine & -18.20295068 & 944.451 & 0 \\
\hline XM_013969000.2 & KAP8 & Keratin 8 & -13.45105267 & 3176.066 & 0.29 \\
\hline NM 001285774.1 & KRTAP3-1 & Keratin associated protein 3-1 & -11.60625424 & 5065.256 & 1.697 \\
\hline XM 013971229.2 & KRTDAP & Keratinocyte differentiation associated protein & -13.34343458 & 2123.409 & 0.216 \\
\hline NM 001285767.1 & KRTAP11-1 & Keratin associated protein $11-1$ & -11.39093149 & 2758.346 & 0.988 \\
\hline XM 018047719.1 & KRT6A & keratin $6 \mathrm{~A}$ & -11.10724961 & 1619.46 & 0.633 \\
\hline XM 018047718.1 & KRT5 & Keratin 5 & -10.78919982 & 12527.875 & 6.214 \\
\hline XM_005693818.3 & KRT14 & Keratin 14 & -10.73352246 & 13575.964 & 7.116 \\
\hline XM 013977322.1 & SPRR4 & Small proline rick protein 4 & -12.11735104 & 838.589 & 0.178 \\
\hline XM 018046116.1 & $\mathrm{TCHH}$ & Trichohyalin & -10.43801428 & 518.08 & 0.321 \\
\hline XM 018063392.1 & KRTDAP & Keratinocyte differentiation associated protein & -11.3513932 & 2312.868 & 1.045 \\
\hline XM 005677520.2 & PRR9 & Proline rich 9 & -10.27471289 & 2590.013 & 1.928 \\
\hline XM 018047715.1 & KRT1 & Keratin 1 & -9.990481309 & 8496.427 & 7.334 \\
\hline XM 005676807.3 & SFN & Stratifin & -10.15748235 & 961.992 & 0.765 \\
\hline XM 018065044.1 & KRT10 & Keratin 10 & -9.733969775 & 7700.574 & 7.99 \\
\hline XM 018047714.1 & KRT77 & Keratin 77 & -9.751593306 & 799.402 & 0.802 \\
\hline NM 001285719.1 & KRT27 & Keratin 27 & -9.574450764 & 2504.223 & 2.952 \\
\hline NM 001285766.1 & KRT25 & Keratin 25 & -9.463088883 & 2720.404 & 3.451 \\
\hline XM 005675022.3 & CSTA & Cystatin-A & -10.93663814 & 715.197 & 0.397 \\
\hline XM_018046124.1 & LOR & Loricin & -9.608742566 & 641.14 & 0.747 \\
\hline XM_005675653.3 & DMKN & Dermokine & -8.890178133 & 695.94 & 1.296 \\
\hline XM 005692309.3 & CST6 & Cystatin-6 & -7.796853214 & 18161.91 & 84.967 \\
\hline XM 018043270.1 & ANXA2 & Annexin A2 & -3.618405089 & 1732.492 & 127.817 \\
\hline XM 018054288.1 & S100A11 & S100 calcium binding protein A11 & -3.280579422 & 1092.421 & 118.984 \\
\hline XM 005677583.3 & PPIA & peptidylprolyl isomerase A & -2.607281603 & 1246.828 & 201.647 \\
\hline XM 018042040.1 & SLC25A4 & solute carrier family 25 member 4 & 6.799276849 & 27.689 & 2777.476 \\
\hline
\end{tabular}

Table 3 detailed the identified LOC symbol genes that are differentially expressed. These are involved in keratinization pathway, keratinocyte differentiation, follicle transition from catagen to telogen phase while rest of LOC symbol genes are uncharacterized and thus are not mentioned and counted here.

Table 2. Comprehensive list of differentially expressed LOC genes in both breed.

\begin{tabular}{|c|c|c|c|c|c|}
\hline Transcript ID & Genes & Gene names & $\operatorname{LogFC}$ & $\begin{array}{l}\text { Pashmina } \\
\text { TMM counts }\end{array}$ & $\begin{array}{l}\text { Barbari } \\
\text { TMM counts }\end{array}$ \\
\hline XM 018065087.1 & LOC108638296 & keratin, high-sulfur matrix protein, B2D-like & -13.09954778 & 1197.751 & 0.136 \\
\hline XM 005693797.3 & LOC102170546 & keratin-associated protein 3-3 & -13.65637106 & 1377.916 & 0.097 \\
\hline XM_018047728.1 & LOC102183211 & keratin, type II cuticular Hb1-like & -11.79556929 & 1148.294 & 0.271 \\
\hline XM 005674695.3 & LOC100861181 & keratin-associated protein $7-1$ & -11.93109531 & 3225.366 & 0.826 \\
\hline XM 005674699.3 & LOC100860930 & keratin-associated protein 6-1 & -13.53441427 & 1517.974 & 0.121 \\
\hline XM_005693813.3 & LOC102176457 & keratin, type I microfibrillar, $47.6 \mathrm{kDa}$-like & -11.50435984 & 1664.06 & 0.511 \\
\hline XM_018065058.1 & LOC102176726 & Keratin, type I microfibrillar $48 \mathrm{kDa}, 8 \mathrm{C}-1$ & -11.33365608 & 1668.517 & 0.576 \\
\hline XM 005677560.3 & LOC102183026 & cornifin-A-like & -11.3070629 & 3227.395 & 1.24 \\
\hline XM_018046520.1 & LOC102187909 & flaggirin-2 & -11.4889734 & 608.103 & 0.192 \\
\hline XM 018065059.1 & LOC102179881 & keratin, type I microfibrillar, $47.6 \mathrm{kDa}$ & -11.15974894 & 1331.023 & 0.518 \\
\hline XM 018065052.1 & LOC102179515 & keratin, type I cytoskeletal 15 & -11.09359986 & 1096.238 & 0.448 \\
\hline XM_005674706.2 & LOC102182256 & keratin-associated protein $26-1$ & -12.2732842 & 779.321 & 0.144 \\
\hline XM_018065090.1 & LOC102177561 & keratin, high-sulfur matrix protein, IIIA3-like & -11.81026409 & 1090.782 & 0.268 \\
\hline NM 001285717.1 & LOC100861381 & Hair acidic keratin 1 & -10.90665807 & 1287.425 & 0.602 \\
\hline XM 018065055.1 & LOC102177275 & keratin, type I cytoskeletal 17 & -10.65089629 & 3005.338 & 1.669 \\
\hline XM 018053338.1 & LOC108636735 & keratin-associated protein 6-1-like & -12.79359006 & 993.626 & 0.135 \\
\hline XM_018065089.1 & LOC108638297 & keratin, high-sulfur matrix protein, $\mathrm{B} 2 \mathrm{C}$ & -11.21869324 & 1432.433 & 0.554 \\
\hline XM_018065091.1 & LOC108638298 & keratin, high-sulfur matrix protein, IIIA3-like & -12.73755675 & 764.901 & 0.132 \\
\hline XM_018047726.1 & LOC102183766 & keratin, type II cuticular $\mathrm{Hb} 1$ & -10.78463873 & 855.636 & 0.433 \\
\hline NM 001285770.1 & LOC100861175 & keratin associated protein 13.1 & -11.3240876 & 1700.814 & 0.692 \\
\hline XM_013962365.2 & LOC102183026 & cornifin-A-like & -15.9313294 & 577.511 & 0 \\
\hline XM_018065093.1 & LOC108638300 & keratin, high-sulfur matrix protein, IIIA3-like & -11.42757254 & 865.616 & 0.308 \\
\hline XM 005674703.3 & LOC102181431 & keratin-associated protein $13-1$ & -11.66937062 & 516.926 & 0.146 \\
\hline XM_018065079.1 & LOC108638291 & keratin, high-sulfur matrix protein, B2D-like & -11.0776741 & 599.149 & 0.287 \\
\hline XM_018047727.1 & LOC102185436 & keratin, type II microfibrillar, component 7C & -10.0443469 & 1290.057 & 1.09 \\
\hline
\end{tabular}




\begin{tabular}{|l|l|l|l|l|l|}
\hline XM_005693796.3 & LOC102170264 & keratin, high sulfur matrix protein, IIIB3 & -10.21592398 & 1079.87 & 0.905 \\
\hline XM_005688097.3 & LOC102175311 & calmodulin & -9.90827306 & 1082.197 & 1.086 \\
\hline XM_018047717.1 & LOC102177231 & keratin, type II cytoskeletal 71 & -9.108103679 & 2887.431 & 4.589 \\
\hline XM_005679937.3 & LOC102184223 & keratin, type II microfibrillar, component 5 & -8.432753572 & 539.167 & 1.367 \\
\hline XM_005692259.2 & LOC102169125 & major allergen I polypeptide chain 2 & -8.304047277 & 1587.193 & 5.515 \\
\hline XM_018062299.1 & LOC106503120 & major allergen I polypeptide chain 1 & -7.835300928 & 880.146 & 4.487 \\
\hline XM_005675654.3 & LOC102186806 & cystatin-B & -3.575714584 & 1002.589 & 87.747 \\
\hline
\end{tabular}

* [14] refers to the gene names included in this table.

\section{Discussion}

To investigate the genes that are responsible for wool/cashmere production, we performed the differential expression analysis on the transcriptome profile of skin samples from two goat breeds, Pashmina and Barbari. Pashmina goat provides protection against the cold harsh environment via its luxurious fiber while Barbari goat breed inhabits the hot arid environment and are valued for meat and milk yield $[4,5]$. The top most DETs were then functionally annotated to get molecular insight of its corresponding genes.

Gene ontology terms of highly expressed HF transcripts in Pashmina and Barbari goat breed revealed that most of the genes are related to keratin differentiation and are involved in keratinization pathways. Several scientific evidence is available and many studies report the genes concurrent to our results. It is previously suggested that keratins (KRT) and Keratin associated proteins (KRTAPs) are the main structural component of hair fibers and sheath [15]. The interaction of fibrous KRTs and matrix KRTAPs are the foundation of cornified skin appendages such as hairs, horns. An analysis on Chinese Tan sheep's skin transcriptome revealed the potential genes for curly fleece and hair/wool formation including KRT27, PRR9 and CST6 that ranged from 3,353 to 7,813 FPKM reads [16]. Another study reported the high expression of $S F N$ gene in Changthangi goat [15]. This Stratifin gene regulates the epithelial keratinization by directly regulating the cell cycle. It has been best selected for human dermal papilla cells (DPCs) as well. Similarly, they analyzed CSTA and KRT77 gene having fold change values of 4.63 and 3.46 respectively that helps in keratinocyte differentiation. A recent analysis on differential expression in Canine anagen, telogen HFs and interfollicular epidermis (IFE) [13] presented that hair follicle associated structural genes (TCHH, KRT25) have high expression levels 3,997.42 and 10,456.27 during anagen phase. Similarly, KRT1, KRT5, KRT10, KRT14 and LOR are epidermal structure-associated genes and have high TPM counts $(21,712.07,12,733.06$, $33,847.68,29,472.32$ and 7229.10) in IFE samples. RNA-seq analyses on Ovis aries revealed S10011A gene association with hair/wool development in them [17]. Small proline-rich protein 4 that has TMM count $>500$ in our Pashmina breed is a class of cornified envelope and are found around $300 \mathrm{~kb}$ of epidermal differentiation complex. An analysis on sheep found its expression in HFs, epidermis and capillaries [18].

Importantly, the LOC symbol genes were also targeted in this study with known functions. A little is known about them but they have been characterized and are previously reported. A study elucidated the role of these LOC symbol genes in transition of HFs from catagen to telogen phase/telogen induction and their differential expression in either phase with FPKM, $\log 2(\mathrm{FC})$ and P-values [19]. Other uncharacterized proteins such as LOC108635855 encoded by XR_001917982.1, LOC108635656 (XR_001917911.1) and many others were also divulged in Pashmina breed with high expression counts but they are not included here.

In conclusion, Pashmina and Barbari breed's skin transcriptome sample were subjected to differential expression analysis to search out potential genes related to HFs. Differential expression patterns above a significant normalized count in the two breeds under consideration suggested the involvement of those genes in keratinization pathways, keratinocyte differentiation, sulfur matrix proteins, dermal papilla cells, HFs proliferation, hair curvature, wool fiber diameter, hair transition, hair shaft differentiation and hair follicle keratinization. All DETs identified in this study can be biologically evaluated in future for pashmina/cashmere fiber evolvement in animals and provides some possible clues for further investigations on gene enrichment analysis of hair development genes. 


\section{References}

1. Fan $Y$, Wu R, Qiao X, Zhang Y, Wang R, Su R, et al. Hair follicle transcriptome profiles during the transition from anagen to catagen in Cashmere goat (Capra hircus). Genet Mol Res 2015; 14 (4): 17904-15.

2. Galbraith H. Fundamental hair follicle biology and fine fiber production in animals. animal 2010; 4 (9): 1490-509.

3. Ryder ML. The use of goat hair: an introductory historical review: Anthropozoologica; 1993.

4. Malik I, Wani F. Pashmina goat farming in cold arid desert of Ladakh: A geographical study of Changthang region. International Journal of Advanced Scientific Research and Management 2017; 2: 1-10.

5. Porter V, Alderson L, Hall SJ, Sponenberg DP. Mason's World Encyclopedia of Livestock Breeds and Breeding, 2 Volume Pack: Cabi; 2016.

6. shutterstock-Pashmina goat images. Available from: https://www.shutterstock.com/search/pashmina+goat.

7. Domestic Animal Breeds. Available from: https://domesticanimalbreeds.com/barbari-goatbreed-everything-you-need-to-know/.

8. Brown J, Pirrung M, McCue LA. FQC Dashboard: integrates FastQC results into a web-based, interactive, and extensible FASTQ quality control tool. Bioinformatics 2017; 33 (19): 3137-9.

9. Bolger AM, Lohse $M$, Usadel B. Trimmomatic: a flexible trimmer for Illumina sequence data. Bioinformatics 2014; 30 (15): 2114-20.

10. Patro R, Duggal G, Love MI, Irizarry RA, Kingsford C. Salmon provides fast and bias-aware quantification of transcript expression. Nature methods 2017; 14 (4): 417-9.

11. Haas BJ, Papanicolaou A, Yassour M, Grabherr M, Blood PD, Bowden J, et al. De novo transcript sequence reconstruction from RNA-seq using the Trinity platform for reference generation and analysis. Nature protocols 2013; 8 (8): 1494-512.

12. Henschel R, Lieber M, Wu L-S, Nista PM, Haas BJ, LeDuc RD, editors. Trinity RNA-Seq assembler performance optimization. Proceedings of the 1st Conference of the Extreme Science and Engineering Discovery Environment: Bridging from the extreme to the campus and beyond; 2012.

13. Wiener DJ, Groch KR, Brunner MA, Leeb T, Jagannathan V, Welle MM. Transcriptome Profiling and Differential Gene Expression in Canine Microdissected Anagen and Telogen Hair Follicles and Interfollicular Epidermis. Genes 2020; 11 (8): 884.

14. National Library of Medicine-Center for Biotechnology Information Search database. Available from: https://www.ncbi.nlm.nih.gov/search/.

15. Ahlawat S, Arora R, Sharma R, Sharma U, Kaur M, Kumar A, et al. Skin transcriptome profiling of Changthangi goats highlights the relevance of genes involved in Pashmina production. Scientific reports 2020; 10 (1): 1-10.

16. Kang X, Liu G, Liu Y, Xu Q, Zhang M, Fang M. Transcriptome profile at different physiological stages reveals potential mode for curly fleece in Chinese tan sheep. PloS one 2013; 8 (8): e71763.

17. Zlobin AS, Volkova NA, Borodin PM, Aksenovich TI, Tsepilov YA. Recent advances in understanding genetic variants associated with growth, carcass and meat productivity traits in sheep (Ovis aries): an update. Archives animal breeding 2019; 62 (2): 579-83.

18. Jinshan Z, Huaiyuan Q, Jingjing X, Liu N, Rongwei H, Perez-Campo F, et al. Discovery of genes and proteins possibly regulating mean wool fiber diameter using CDNA microarray and proteomic approaches. Scientific Reports (Nature Publisher Group) 2020; 10 (1). 
19. Follicle transition from Anagen to Catagen / Catagen Induction. Available from: http://Ims.snu.edu.in/pidb/pashmina_cycling/ana_cat.php. 